\title{
Pesquisas em Psicoterapia: Seções Especiais de Periódico (1995 a 2005) ${ }^{1}$
}

\author{
Maria Leonor Espinosa Enéas ${ }^{2}$ \\ Universidade Presbiteriana Mackenzie
}

\begin{abstract}
RESUMO - O artigo apresenta pesquisas em psicoterapia para completar uma visão qualitativa das tendências da área. Analisa sete seções especiais sobre o tema publicadas no período de 1995 a 2005 em um conceituado periódico da American Psychological Association (APA). Verifica que as grandes ênfases da área - busca de evidência científica e articulação da pesquisa com a prática clínica - permanecem no foco das discussões, principalmente o debate sobre os ensaios clínicos controlados. Constata produção menos intensa no período, mas condições mais objetivas de uma possível aproximação entre os pesquisadores e os clínicos. Os desafios persistem, agora buscando novos caminhos.
\end{abstract}

Palavras-chave: processo psicoterápico; produção científica; análise documental; revisão de literatura.

\section{Psychotherapy Researches: Special Sections of a Journal (1995 to 2005)}

\begin{abstract}
The article presents psychotherapy research to complete a qualitative view of the tendencies in this field. Seven special sections about this issue published between 1995 and 2005 in an important journal of the American Psychological Association (APA) are analyzed. It verifies that the great emphases in the field - the search for scientific evidence and the combination of the research and clinical practice - are still the focus of the discussions, mainly the debate about the controlled clinical essays. Less intensive production in the period but more objective conditions to a possible approach between the researchers and the clinicians are observed. There are still challenges, now searching new directions.Key words: identification; psychoanalysis; metapsychology.
\end{abstract}

Key words: psychotherapeutic process; scientific production; documental analysis; literature review.

Este artigo se propõe a acompanhar o desenvolvimento das pesquisas em psicoterapia por meio da análise de seções especiais do Journal of Consulting and Clinical Psychology (JCCP) entre os anos de 1995 a 2005 por meio do recorte das seções especiais que constituem os destaques de maior interesse ou importância no momento de sua publicação (Garfield, 1981). A proposta é abranger um período amplo de publicações dessas seções especiais pois a área de pesquisa em psicoterapia, mesmo em crescente desenvolvimento, ainda não apresenta a maturidade de outras áreas científicas cuja produção deveria ser avaliada mais amiúde (Witter, 1999). Uma característica importante do JCCP é apresentar estudos que seguem rigorosos métodos de pesquisa clínica (Newman \& Howard, 1991). Isso o torna um periódico de ponta em pesquisas em psicoterapia (Borkenhagen, Decker, Brähler \& Strauss, 2002) e, portanto, uma fonte significativa para a presente análise. Com esse recorte para análise qualitativa, pretende-se contribuir para uma reflexão quanto ao desenvolvimento das pesquisas a partir da produção de

1 Parte deste trabalho foi apresentada na Introdução da Tese de Doutorado defendida na PUCCAMP em abril de 1999 sob orientação da Dra. Elisa Medici Pizão Yoshida. Dados atualizados foram apresentados em Mesa Redonda no Congresso da ULAPSI em abril de 2005 e completados para o presente artigo

2 Endereço: Clínica psicológica, Rua Maria Antonia, 358, $1^{\circ}$ andar, São Paulo, SP, Brasil 01222-010. E-mail: mleeneas@ mackenzie.com.br centro mais avançado que conta com maior sustentação empírica. Tal análise pode inspirar propostas de pesquisa que auxiliem a responder os mesmos questionamentos em nosso meio.

\section{Método de seleção e análise das seções especiais}

Foram identificados os temas destacados no JCCP no período entre 1995 e 2005. Tais temas serão referidos como seções especiais, nome genérico e mais amplamente usado no periódico. Todas as seções são compostas predominantemente por estudos empíricos e, aquelas aqui analisadas que se referem a psicoterapia, são relativas a processos terapêuticos realizados com indivíduos adultos. Também foram incluídos temas conexos referentes a aspectos relevantes para a área e que são apontados e discutidos nas seções mais específicas.

Verifica-se na Tabela 1 a irregularidade na publicação dessas seções, com decréscimo ao longo do tempo. Relativamente ao interesse por pesquisa em psicoterapia, sua publicação mais significativa ocorreu no início do período mas persistiu até 2001, quando ocorreu a última seção observada.

Das 22 seções especiais publicadas nesses 11 anos, cinco referem-se a pesquisa em psicoterapia e duas a temas conexos, conforme a Tabela 1. Outras áreas com destaque referem-se ao tratamento de crianças e adolescentes - não aparecendo mais nada relativo a casais e família - $(N=4)$, os 
Tabela 1. Distribuição do número de seções especiais (Esp.) do JCCP e do número de seções especiais referentes à pesquisa em psicoterapia (Pesq.), incluindo as seções de temas conexos $(*)$ por ano considerado.

\begin{tabular}{lccccc}
\hline Ano & Esp. & Pesq. & Ano & Esp. & Pesq. \\
\hline 1995 & 5 & 1 & 2001 & 2 & 1 \\
1996 & 7 & $2(*)$ & 2002 & 3 & $\mathrm{xx}$ \\
1997 & 1 & $\mathrm{xx}$ & 2003 & $\mathrm{xx}$ & $\mathrm{xx}$ \\
1998 & 1 & 1 & 2004 & $\mathrm{xx}$ & $\mathrm{xx}$ \\
1999 & 1 & $1(*)$ & 2005 & 1 & $\mathrm{xx}$ \\
2000 & 1 & 1 & & & \\
\hline
\end{tabular}

temas médicos e de treinamento e tratamento tiveram duas seções cada um. Nesse período observa-se uma diminuição a menos da metade do montante das seções publicadas no período analisado anteriormente ( $N=46$ entre 1981 e 1994, Enéas, no prelo). A diminuição mais significativa com o tema ocorreu com transtornos ou situações específicas que teve uma seção especial ainda no início do período considerado e contava com 16 no período anterior.

Tabela 2. Distribuição das seções especiais sobre pesquisa em psicoterapia por ano, editor e tema, incluindo as seções de temas conexos (*).

\begin{tabular}{lll}
\hline Ano & Editor & Tema \\
\hline 1995 & Russell & $\begin{array}{l}\text { Pesquisa multivariada de } \\
\text { processo em psicoterapia. }\end{array}$ \\
1996 & Greenberg e Newman & $\begin{array}{l}\text { Uma abordagem para pesquisa } \\
\text { de processo de mudança em } \\
\text { psicoterapia. }\end{array}$ \\
1996 & Benjamin & $\begin{array}{l}\text { Análise Estrutural do } \\
\text { Comportamento Social. * }\end{array}$ \\
1998 & Kendall & $\begin{array}{l}\text { Terapias psicológicas } \\
\text { empiricamente sustentadas. }\end{array}$ \\
1999 & Kendall & Significância clínica. * \\
2000 & Haaga & $\begin{array}{l}\text { Modelo de cuidados } \\
\text { escalonados em psicoterapia. }\end{array}$ \\
& Lambert & $\begin{array}{l}\text { Pesquisa focalizada no } \\
\text { paciente. }\end{array}$ \\
\hline
\end{tabular}

Foram analisadas sete seções, sendo cinco relativas a pesquisa em psicoterapia propriamente dita e duas a temas conexos, conforme a Tabela 2 . O procedimento de levantar e comentar os principais aspectos discutidos nestas seções, mais especialmente as tendências e perspectivas observadas foi usado em artigo anterior referente ao período entre 1981 e 1994 (Enéas, no prelo) e foi mantido para este artigo para oferecer um panorama sistematizado sobre o tema, dado que estas seções apresentam os destaques do momento de sua publicação.

As seções serão apresentadas cronologicamente e seguidas de comentários que articulem a contribuição do período. Esta será comparada com as tendências verificadas no período anterior.

\section{Análise das Seções}

Russell (1995) introduz seção especial sobre pesquisa multivariada de processo em psicoterapia, que enfoca estrutura e mudança em sintomas e aspectos do discurso do paciente e também do terapeuta durante o tratamento, empregando métodos de análise fatorial. Esse enfoque dual sobre a estrutura e a seqüência do processo torna esses estudos diferentes dos anteriormente realizados (Enéas, no prelo), procurando explorar realmente a essência do processo que o autor considera inexplorado pelos métodos tradicionais. Um exemplo é o estudo de Luborsky (1995), que revisa seu artigo de 1953 e no qual faz mensurações longitudinais sobre inúmeras categorias de variáveis do mesmo paciente, procurando identificar quais fatores variam juntos no decorrer do tempo. Essas variáveis incluem aspectos fisiológicos e psicológicos. Contudo, como salienta Luborsky, é necessária a habilidade clínica para dar sentido desde a seleção das variáveis até a compreensão do contexto dos sintomas. Stiles e Shapiro (1995) verificam o agrupamento das respostas para identificar padrões e acompanhar a evolução de uma terapia bem-sucedida. Czogalik e Russell (1995) apresentam a comparação gráfica das interações ocorridas no terço médio de dois processos terapêuticos, um bem-sucedido e outro mal-sucedido, acompanhada de interpretação clínica das transcrições. Supõem que essa representação seja mais sensível para revelar diferenças entre os casos do que a comparação quantitativa de médias. Observando essa seção, comparativamente à de 1993 (Enéas, no prelo) sobre os fatores curativos em psicoterapia dinâmica, nota-se que os avanços ocorridos referem-se a questões parciais do processo terapêutico; lá, o refinamento conceitual dos fatores curativos, aqui, o estudo do discurso de ambos os participantes.

Em 1996, mais duas seções especiais. A primeira delas, introduzida por Greenberg e Newman, discute uma abordagem para pesquisa de processo de mudança em psicoterapia. Os autores comentam a dupla finalidade da seção, que procura enfocar pesquisa de mudança em psicoterapia e explicar uma abordagem para desenvolver um programa de pesquisa envolvendo oito passos baseados nos princípios de decomposição e localização desenvolvidos por Greenberg. Nessa abordagem, os primeiros passos incluem uma exploração inicial do material para evidenciar os comportamentos significativos. Os autores colocam em questão a importância de valorizar os estudos sobre esses primeiros passos, incluindo sua publicação em periódicos. Sua proposta é de construir um modelo de mini teorias sobre o desenrolar do processo e os padrões de interação que nele ocorrem para serem testados em sua validade preditiva. Os artigos da seção discutem aliança terapêutica (Safran \& Muran, 1996; Sexton, Hembre \& Kvarme, 1996), vivacidade das expressões do paciente (Watson, 1996), atribuição de sentido aos eventos críticos de mudança vividos emocionalmente pelo paciente (Clarke, 1996) e interpretação (Joyce \& Piper, 1996). No artigo de Kolden (1996) são definidos processos de mudança universais, geralmente transteoréticos e comuns aos diversos modelos de terapia, e os específicos, conceitualizados em um nível microscópico de análise. São identificados cinco processos de mudança 
universais: contato terapêutico, operações terapêuticas, vínculo terapêutico, abertura e envolvimento terapêutico e realizações terapêuticas.

A segunda seção do ano, apresentada por Benjamin (1996), refere-se à análise estrutural do comportamento social (SASB). Esse modelo, originalmente ateórico, procura dissecar eventos interpessoais e intrapsíquicos em três dimensões subjacentes: foco, afiliação e interdependência, e permite operacionalizar aspectos importantes de uma ampla série de eventos psicológicos, gerando inclusive códigos de comportamento em contextos específicos. Trata-se, portanto, de um tema de interesse conexo. Connolly e cols (1996) discutem variações de padrões de transferências em psicoterapia empregando narrativas aleatoriamente escolhidas entre a amostra avaliada. Ainda assim discutem a dificuldade para avaliar a significância estatística em função de os dados não serem baseados em pressupostos de independência e normalidade. O estudo de Henry (1996) procura operacionalizar conceitos psicodinâmicos, o que geralmente constitui uma tarefa difícil em função do alto nível de abstração que tais conceitos empregam. O autor comenta que a congruência problema-tratamento-resultado deve-se à conceitualização e medida coerentes entre os problemas do paciente, o processo de mudança terapêutica e os resultados clínicos.

Kendall (1998) apresenta a seção especial sobre terapias psicológicas empiricamente sustentadas, movimento de terapias que volta-se para a definição de um procedimento que conduza a mudanças atestadas e não meramente casuais ou dependentes de características individuais de um condutor do processo. Dessa forma, para documentar que a mudança não acontece por acaso, a significância estatística deve preceder a significância clínica. Chambless e Hollon (1998) mencionam que essas terapias têm como questões mais importantes a eficácia (que inclui significância clínica), a efetividade (ou utilidade clínica) e a eficiência (ou custo-efetividade). Para responder às questões de eficácia, propõem o uso de delineamentos de pesquisa cuidadosamente controlados que devem ser replicados. Algumas questões revelam-se mais complexas, como a da significância clínica, que ainda não conta com um nível específico bem estabelecido, principalmente pela dificuldade de aferir quanta mudança é requerida para um determinado problema devido à pouca disponibilidade de dados referentes ao tema. Quanto à efetividade, os delineamentos empregados devem captar a "completa riqueza e variabilidade da real prática clínica" (p.14); os autores reconhecem que os ensaios clínicos controlados não podem fazê-lo, pois a randomização necessária ao método afeta a representatividade do encontro terapêutico. Assim, muitas vezes, métodos observacionais são melhor aplicados. Dentro do aspecto da eficiência, Beutler (1998) aponta a quase imposição para a realização de tratamentos empiricamente sustentados, uma vez que o ambiente político em que se encontram exige que sejam tomadas medidas nessa direção.

Ainda nessa seção, Garfield (1998), comentando a variabilidade da prática clínica diz que, a despeito do que é conhecido, ainda é necessário que "aprendamos muito mais sobre os ingredientes ou fatores ativos nas terapias que levem a uma mudança positiva antes que possamos realmente compreender o processo terapêutico" (p.123). Persons e Silberschatz (1998) discutem a utilidade dos resultados desses estudos para os psicoterapeutas. Por seu lado, Persons defende seu emprego por razões clínicas, éticas e legais, enfatizando sua importância para estudos de resultado. De outro lado, Silberschatz considera tais estudos inúteis aos clínicos pois só respondem às questões de eficácia, além de proporem a manualização, que é um requisito questionável, por ser de difícil aderência e por essa aderência nem sempre estar associada à experiência no atendimento. Para ele, essas pesquisas sacrificam a validade clínica em benefício da maximização do controle experimental. $\mathrm{O}$ autor enfatiza que é praticamente impossível considerar iguais fenômenos tão complexos e multidimensionais como a psicopatologia e a psicoterapia, além disso, é impossível, segundo ele, tornar iguais as situações terapêuticas, não importando o montante de manipulação experimental. Continuando a seção, Goldfried e Wolfe (1998) criticam os artigos aí apresentados por considerá-los metodologicamente elegantes mas falhos quanto à validade clínica. Reconhecem que o direcionamento das pesquisas segue pressões das agências governamentais e de seguro, porém temem que as restrições de ordem metodológica sobre os delineamentos de pesquisa possam ter conseqüências restritivas sobre a prática clínica. Comentam ainda que esses estudos refletem a posição da terceira geração de pesquisa, que tende a seguir o modelo médico de pesquisa com medicação; defendem, no entanto, a necessidade de buscar uma quarta geração, ainda não bem definida, que inclua, entre outras coisas, a colaboração entre pesquisador e clínico, além de estudos de tratamentos teoricamente integrados. Essa reação parece ser o ápice da ênfase sobre a busca de refinamento metodológico e da discussão quanto à insatisfação causada pela dificuldade de atingir uma aproximação mais satisfatória com o objeto de estudo.

As dificuldades e polêmicas relativas à significância clínica culminam em uma seção sobre esse tema conexo em 1999, que apresenta as atuais metodologias, estratégias conceituais e considerações avaliativas. Kendall (1999) salienta na introdução que, como o campo da saúde mental se dirige para uma melhor compreensão dos tratamentos psicossociais que possam ser ditos eficazes, é preciso verificar o grau em que esses tratamentos, que se mostraram bem-sucedidos na redução de sintomas, também sejam clinicamente significativos. Kendall, Mars-Garcia, Nath e Sheldrick (1999) apresentam uma abordagem descritiva para avaliar significância clínica por meio de comparações normativas, sendo necessário que os pesquisadores definam as variações de comportamento consideradas como "normativas". Apontam problemas como: a definição de qual população deve ser considerada normativa, a possibilidade de para cada questão de pesquisa haver uma população normativa específica e a equivalência clínica entre os níveis pós-tratamento e normativos relativos a uma variável não ser necessariamente generalizável para outras variáveis. Jacobson, Roberts, Berns e McGlinchey (1999) definem a mudança clinicamente significativa como o retorno ao funcionamento normal, sendo importante considerar que a magnitude da mudança para um dado indivíduo deve ser estatisticamente confiável, ou seja, ir além do escopo daquilo 
que poderia razoavelmente ser atribuído ao acaso ou ao erro de medida. Foster e Mash (1999) argumentam, entre outras coisas que, embora discutida como um construto único e dicotômico, constitui um construto multidimensional que engloba pelo menos dois outros construtos gerais - aceitabilidade e importância - que também são multidimensionais e dinâmicos. Novamente a constatação de que, apesar dos avanços representarem refinamentos nas medidas, as necessidades de maior precisão persistem, ainda muito ligadas à própria complexidade do objeto de estudo.

Nesse período observa-se o desenvolvimento nas propostas de pesquisa conseguindo maior aproximação com a relação entre as variáveis e com o contexto dos processos, embora ainda sejam apontados refinamentos necessários à área. No centro da polêmica pela busca de eficácia, os ensaios clínicos controlados dividem os pesquisadores entre seus defensores - em nome da evidência científica - e seus críticos, que não vêem sentido em resultados obtidos com tanto controle que torna o contexto artificial e, portanto, sem utilidade clínica. A discussão desse delineamento envolve o aspecto da significância clínica e da significância estatística, esta última priorizada pelas terapias empiricamente sustentadas. No tocante à significância clínica, são apontados avanços relativos aos apresentados em 1991 e também as dificuldades que persistem na área.

Em 2000 foi a vez do modelo de cuidados escalonados em psicoterapia, voltado para a abordagem comportamental. Haaga (2000) comenta na Introdução que os modelos de cuidados escalonados representam uma tentativa de maximizar a eficiência da alocação de recursos em psicoterapia, sendo as intervenções de custo mais baixo tentadas primeiro e as intervenções mais intensivas e custosas reservadas para aqueles que forem insuficientemente ajudados pelas intervenções iniciais. $\mathrm{O}$ autor sugere que as avaliações prétratamento poderiam ser usadas para equiparar os pacientes com a intensidade apropriada e o tipo de intervenção com a base das ligações de avaliação de tratamento substanciadas empiricamente. Desafortunadamente, como notado por vários autores nessa seção especial, tais regras de decisão empírica são usualmente falhas e necessitam de pesquisas rigorosas sobre esquemas de alocação de recursos de tratamento. Dentro da perspectiva de emprego de intervenções de baixo custo encontram-se medidas de aprendizagem de técnicas de terapia que não necessitem da presença do terapeuta como videotapes, métodos de auto-ajuda, entre outros que, como sugere Newman (2000), podem incluir pacientes que são tipicamente excluídos dos ensaios clínicos randomizados ou aqueles que são resistentes a esses tratamentos. As propostas pretendem, como salienta Davison (2000), fazer mais com menos, ou seja, atender a um maior número de pessoas sendo possível denotar menos tempo a cada uma. São propostos modelos de tratamento para transtorno de ansiedade (Newman, 2000), transtorno de pânico (Otto, Pollack \& Maki, 2000), transtornos alimentares (Wilson, Vitousek \& Loeb, 2000) e problemas com álcool (Sobell \& Sobell, 2000).

No ano seguinte, a seção sobre pesquisa focalizada no paciente é introduzida por Lambert (2001), que comenta ser uma área com crescente número de pesquisas e que pode ser distinta da tradicional pesquisa de resultado de terapia por ser focalizada no paciente individual e não nos resultados de grupos de pacientes. Lambert aponta que as pesquisas de efetividade estreitam a lacuna entre a prática clínica e a pesquisa, mas ainda não respondem às maiores preocupações cotidianas dos clínicos. A pesquisa focalizada no paciente pode ser mais facilmente realizável pelos clínicos em sua prática diária e permite predições do progresso do paciente e a modificação dos planos de tratamento em virtude do monitoramento constante dos indivíduos. Nessa metodologia, os dados da curva de melhora individuais são usados para gerar curvas de expectativas de melhora para pacientes semelhantes. Além disso, foram desenvolvidos métodos estatísticos para discernir mudança confiável e clinicamente significativa. Dentro desse contexto, a seção especial apresenta quatro programas de pesquisa que usam a pesquisa focalizada no paciente especificamente designada para promover a qualidade do cuidado do paciente. A título de exemplo, o artigo de Lueger e cols (2001) apresenta um trabalho pioneiro na área que pretende a garantia de qualidade de forma a identificar a equiparação mais eficiente entre o problema, paciente, terapia e terapeuta, cujo objetivo último é selecionar o melhor tratamento para um paciente em particular. Lambert, Hansen e Finch (2001) apresentam seu modelo de trabalho que pretende ser um complemento a cuidadosos ensaios clínicos. Enfatizam a importância do conceito e da operacionalização da mudança clinicamente significativa para o desenvolvimento das pesquisas focalizadas no paciente visto que, quanto ao delineamento do tratamento para um paciente, é preciso um marcador para definir as decisões sobre a necessidade de mais tratamento, término ou encaminhamento.

A verificação de Omer e Dar (1992) de que as pesquisas se tornaram mais pragmáticas mostra uma tendência que se manteve também nos anos seguintes. Parece que começa a haver uma conjunção mais explícita das principais tendências já observadas nos anos anteriores (Enéas, no prelo) - de buscar evidência científica e de articular a pesquisa à prática clínica - e também uma perspectiva mais objetiva de que os avanços necessários possam ser alcançados. Talvez possa ser configurada, assim, uma nova geração de pesquisa, como sugerem Goldfried e Wolfe (1998), em que os pesquisadores possam trabalhar em conjunto com os clínicos e se possa avançar tanto nas pesquisas quanto nos próprios tratamentos.

\section{Comentários Finais}

Como observado, as grandes preocupações da área de pesquisa em psicoterapia, já evidentes no período anterior (Enéas, no prelo) - busca de evidência científica e necessidade de articulação entre a pesquisa e a prática clínica - continuam sendo bastante enfatizadas nesse período. No tocante à primeira, grande polêmica cercou a necessidade de evidência científica para as psicoterapias, principalmente com os ensaios clínicos randomizados. As pressões para o desenvolvimento de ensaios clínicos controlados foram aumentadas com a publicação do Relatório do Consumidor (Consumer Reports) em 1995 (VandenBos, 1996). Em 1996, uma seção especial no American Psychologist evidenciava, em síntese, a importância sentida em considerar a opinião 
pública quanto à eficácia dos tratamentos oferecidos (Barlow, 1996; Newman \& Tejeda, 1996). Contudo, na opinião de Seligman (1996), há falhas metodológicas no estudo do Relatório do Consumidor induzindo a conclusões que podem servir apenas às necessidades de diminuir os gastos com o atendimento à saúde mental - tendência evidente na seção especial de 2000 -, com prejuízos tanto para o desenvolvimento do conhecimento em psicoterapia quanto para o próprio atendimento da população. Nessa mesma seção, Howard, Moras, Brill, Martinovich e Lutz (1996), discutindo formas de aprimoramento das pesquisas, induzem a pesquisa focalizada no paciente como um novo paradigma para avaliar a psicoterapia monitorando o progresso obtido pelo paciente no decorrer do processo. Essa sugestão cristalizou-se em tendência observada na seção especial de 2001 que parece tentar uma aproximação entre estudos de eficácia e efetividade.

Embora algum progresso tenha sido verificado, principalmente no tocante às pesquisas de caso único e aos aspectos mais parciais da interação terapeuta-paciente, ainda há muitas críticas relativas às questões de desenvolvimento metodológico e estatístico. Houve um aprimoramento na noção de $o$ que e como deveria ser investigado em um processo terapêutico, resultando também no refinamento de sua definição. As interações paciente-terapeuta abrangem uma rede de variáveis relacionadas que se constituem como sub-processos (moleculares) inseridos em um processo (molar) que, por sua vez, também se insere num contexto mais amplo (chamado ecológico por Anderson \& Strupp, 1996), passível de interferir nas interações do processo. Os principais avanços verificados no campo referem-se ao desenvolvimento de modos de considerar o contexto das complexas interações terapêuticas e empregar escalas de avaliação de processo que possam dar credibilidade científica ao conhecimento na área, além de procurar captar o progresso dos fatores curativos em psicoterapia.

Outra questão polêmica é da aplicabilidade dos métodos estatísticos, que requer cuidadosa atenção por parte dos pesquisadores. Dar, Serlin e Omer (1994), em uma revisão que abrangeu três décadas de pesquisa em psicoterapia, verificaram crescentes falhas no emprego de tratamentos estatísticos dos dados de pesquisa. Para os autores, tais falhas se ligam à tendência verificada em estudo anterior (Omer \& Dar, 1992) referente ao mesmo período, de que as pesquisas passaram a ser mais pragmáticas e menos teóricas. ClarkCarter (1997), em revisão de artigos recentes publicados no British Journal of Psychology, ressalta que não está sendo considerado o poder dos testes estatísticos quando de seu emprego, mantendo um risco de os pesquisadores rejeitarem suas hipóteses de pesquisa. Dessa forma, os modelos de tratamento estatístico carecem de mais desenvolvimento e melhor aplicabilidade.

Um avanço importante refere-se às medidas de significância clínica - que serviriam para responder ao objetivo da pesquisa clínica - com melhores métodos para sua determinação, mas que ainda requerem maiores refinamentos.

No tocante à segunda grande preocupação - a distância entre a prática clínica e a metodologia de pesquisa - Persons (1991) atribui esse descompasso ao fato de as pesquisas de resultado serem padronizadas, ateóricas e separarem diagnóstico e tratamento. Houve também uma seção especial no Journal of Clinical Psychology em 1998 para discutir esse tema da utilidade clínica das pesquisas. Beutler e Howard (1998) - na introdução - consideram que as pesquisas podem ser úteis à clínica na medida em que gerem conhecimento que possa ser diretamente usado para melhorar a prática clínica. Já Fernández-Alvarez, Garcia e Scherb (1998) apontam dois tipos de dificuldades na realização de pesquisas no contexto clínico: qual seria o valor de estudos de caso quando comparados ao método experimental tradicional e o quanto a pesquisa pode ser controlada e sistematizada, sendo feita nesse contexto. Dessa forma, propõem uma espécie de conciliação entre a atitude crítica do pesquisador e a responsabilidade do terapeuta em promover alívio aos seus pacientes. Observa-se, assim, que a articulação entre pesquisa e prática clínica perpassa todas as seções em busca de diminuir a distância entre eles, tanto com relação ao emprego de dados de pesquisa pelos clínicos quanto pela dificuldade/possibilidade de os clínicos se envolverem em pesquisa.

Uma outra observação interessante, feita por Beutler (1990), é de que a cisão entre pesquisadores de processo e de resultado se transformou na cisão entre defensores de observações objetivas - definições descritivas - e de dados guiados pela teoria - inferências abstratas. As seções enfocam questões e avanços em ambas as perspectivas.

Como se observou, os assuntos tratados pelas seções especiais refletem o movimento histórico da evolução das pesquisas de processo psicoterápico. Como qualquer história, é melhor conhecida quando contextualizada. No tema em questão, trata-se do ocorrido em um país com grande tradição e desenvolvimento no campo da pesquisa, desenvolvimento esse advindo, entre outros fatores, de necessidades de ordem político-econômica. O conhecimento dessa história tem algo de útil a ensinar, mesmo para locais diferentes, desde que se tenha cautela para interpretar o contexto de inserção dessas pesquisas, bem como das que podem ser realizadas em outros contextos. Sabe-se da necessidade de avançar no conhecimento da funcionalidade - verificação de eficácia e efetividade - dos processos psicoterápicos, tal qual empregados na prática. As pesquisas de processo terapêutico em nosso meio, por exemplo, ainda engatinham, mas o avanço metodológico alcançado em contextos mais evoluídos, bem como a consideração crítica daquilo que se revelou mais importante nesse percurso, podem permitir uma adaptação às reais condições aqui vividas. Ainda é preciso saber quais as possibilidades de ajudar os pacientes, com sua demanda tão grandemente diversificada, em suas necessidades emocionais, de forma a corresponder às suas expectativas e condições, minimizando o sofrimento emocional da população em geral. Além disso, não pode ser esquecido o ambiente no qual se realizam as pesquisas e também as psicoterapias, com suas condições desafiadoras. Para tanto, conhecer e observar como outros grupos procuram superar suas dificuldades, principalmente, como aponta Santeiro (2005), em país com maior tradição em pesquisas clínicas com grandes centros responsáveis por firme sustentação empírica, pode levar a passos que agilizem a busca de nossas próprias soluções. Sem esquecer que a busca de solução científica, como bem 
lembra Fonagy (2005), precisa ser cautelosa e ajustada à complexidade da interação humana, que se mostra o grande fator terapêutico.

\section{Referências}

Anderson, T. \& Strupp, H. H. (1996). The ecology of psychotherapy research. Journal of Consulting and Clinical Psychology, 64, 776-782.

Barlow, D. H. (1996). Health care policy, psychotherapy research, and the future of psychotherapy. American Psychologist, 51, 1050-1058.

Benjamin, L. S. (1996). Introduction to the special section on structural analysis of social behavior. Journal of Consulting and Clinical Psychology, 64, 1203-1212.

Beutler, L. E. (1990). Introduction to the special series on advances in psychotherapy process research. Journal of Consulting and clinical Psychology, 58, 263-264.

Beutler, L. E. (1998). Identifying empirically supported treatments: What if we didn't? Journal of Consulting and Clinical Psychology, 66, 113-120.

Beutler, L. E. \& Howard, K. I. (1998). Clinical utility research: an introduction. Journal of Clinical Psychology, 54, 297-301.

Borkenhagen, A., Decker, O., Brähler, E. \& Strauss, B. (2002). The Journal of the Society for Psychotherapy Research and the diversity of Psychotherapy Research: a compilation and comparison of North American and European contributions. Psychotherapy Research, 12(4), 491-514.

Chambless, D. L. \& Hollon, S. D. (1998). Defining empirically supported therapies. Journal of Consulting and Clinical Psychology, 66, 7-18.

Clarke, K. M. (1996). Change processes in a creation of meaning event. Journal of Consulting and Clinical Psychology, 64, 465-470.

Clark-Carter, D. (1997). The account taken of statistical power in research published in the British Journal of Psychology. British Journal of Psychology, 88, 71-83.

Connolly, M. B., Crits-Christoph, P., Demorest, A., Azarian, K., Muenz, L. \& Chittams, J. (1996). Varieties of transference patterns in psychotherapy. Journal of Consulting and Clinical Psychology, 64, 1213-1221.

Czogalik, D. \& Russell, R. L. (1995). Interactional structures of therapist and client participation in adult psychotherapy: $\mathrm{P}$ techinique and chronography. Journal of Consulting and Clinical Psychology, 63, 28-36.

Dar, R., Serlin, R. C. \& Omer, H. (1994). Misuse of statistical tests in three decades of psychotherapy research. Journal of Consulting and Clinical Psychology, 62, 75-82.

Davison, G. C. (2000). Stepped care: doing more with less? Journal of Consulting and Clinical Psychology, 68, 580585.

Enéas, M. L. E. (no prelo). Pesquisas em Psicoterapia: Seções Especiais (1981-1994). Psicologia: Teoria e Pesquisa.

Fernández-Alvarez, H, Garcia, F. S. \& Scherb, E. (1998). The research program at AIGLE. Journal of Clinical Psychology, 54, 343-359.

Fonagy, P. (2005). Estudos sobre a efetividade das psicoterapias. Em C.L. Eizirik, R.W. Aguiar \& S.S. Schestatsky (Eds.), Psicoterapia de orientação analítica: fundamentos teóricos e clínicos. (pp. 424-461). Porto Alegre: Artes Médicas.
Foster, S. L. \& Mash, E. J. (1999). Assessing social validity in clinical treatment research: issues and procedures. Journal of Consulting and Clinical Psychology, 67, 308-319.

Garfield, S. L. (1981). [Nota do editor]. Journal of Consulting and Clinical Psychology, 49, 146.

Garfield, S. L. (1998). Some comments on empirically supported treatments. Journal of Consulting and Clinical Psychology, 66, 121-125.

Goldfried, M. R. \& Wolfe, B. E. (1998). Toward a more clinically valid approach to therapy research. Journal of Consulting and Clinical Psychology, 66, 143-150.

Greenberg, L. S. \& Newman, F. L. (1996). An approach to psychotherapy change process research: introduction to the special section. Journal of Consulting and Clinical Psychology, 64, 435-438.

Haaga, D. A. F. (2000). Introduction to the special section on stepped care models in psychotherapy. Journal of Consulting and Clinical Psychology, 68, 547-548.

Henry, W. P. (1996). Structural analysis of social behavior as a common metric for programmatic psychopathology and psychotherapy research. Journal of Consulting and Clinical Psychology, 64, 1263-1275.

Howard, K. I., Moras, K., Brill, P. L., Martinovich, Z. \& Lutz, W. (1996). Evaluation of psychotherapy: Efficacy, effectiveness, and patient progress. American Psychologist, 51, 1059-1064.

Jacobson, N. S., Roberts, L. J., Berns, S. B. \& McGlinchey, J. B. (1999). Methods for defining and determining the clinical significance of treatment effects: description, application, and alternatives. Journal of Consulting and Clinical Psychology, 67, 300-307.

Joyce, A. S. \& Piper, W. E. (1996). Interpretive work in short-term individual psychotherapy: an analysis using hierarchical linear modeling. Journal of Consulting and Clinical Psychology, 64, 505-512.

Kendall, P. C. (1998). Empirically supported psychological therapies. Journal of Consulting and Clinical Psychology, 66, 3-6.

Kendall, P. C. (1999). Clinical significance. Journal of Consulting and Clinical Psychology, 67, 283-284.

Kendall, P. C., Marrs-Garcia, A., Nath, S. R. \& Sheldrick, R. C. (1999). Normative comparisons for the evaluation of clinical significance. Journal of Consulting and Clinical Psychology, 67, 285-299.

Kolden, G. G. (1996). Change in early sessions of dynamic therapy: universal processes and the generic model of psychotherapy. Journal of Consulting and Clinical Psychology, 64, 489-496.

Lambert, M. J. (2001). Psychotherapy outcome and quality improvement: introduction to the special section on patientfocused research. Journal of Consulting and Clinical Psychology, 69, 147-149.

Lambert, M. J., Hansen, N. B. \& Finch, A. E. (2001). Patient-focused research: using patient outcome data to enhance treatment effects. Journal of Consulting and Clinical Psychology, 69, 159-172.

Luborsky, L. (1995). The first trial of the P technique in psychotherapy research - a still-lively legacy. Journal of Consulting and Clinical Psychology, 63, 6-14.

Lueger, R. J., Howard, K. I., Martinovich, Z., Lutz, W., Anderson, E. E. \& Grissom, G. (2001). Assessing treatment progress of individual patients using expected treatment response models. Journal of Consulting and Clinical Psychology, 69, 150-158. 
Newman, M. G. (2000). Recommendations for a cost-offset model of psychotherapy allocation using generalized anxiety disorder as an example. Journal of Consulting and Clinical Psychology, 68, 549-555.

Newman, F. L. \& Howard, K. I. (1991). Introduction to the special section on seeking new clinical research methods. Journal of Consulting and Clinical Psychology, 59, 8-11.

Newman, F. L. \& Tejeda, M. J. (1996). The need for research that is designed to support decisions in the delivery of mental health services. American Psychologist, 51, 1040-1049.

Omer, H. \& Dar, R. (1992). Changing trends in three decades of psychotherapy research: the flight from theory into pragmatics. Journal of Consulting and Clinical Psychology, 60, 88-93.

Otto, M.W., Pollack, M.H. \& Maki, K.M. (2000). Empirically supported treatments for panic disorder: costs, benefits, and stepped care. Journal of Consulting and Clinical Psychology, 68, 556-563.

Persons, J. B. (1991). Psychotherapy outcome studies do not accurately represent current models of psychotherapy. American Psychologist, 46, 99-106.

Persons, J. B. \& Silberschatz, G. (1998). Are results of randomized controlled trials useful to psychotherapists? Journal of Consulting and Clinical Psychology, 66, 126-135.

Russell, R. L. (1995). Introduction to the special section on multivariate psychotherapy process research: structure and change in the talking cure. Journal of Consulting and Clinical Psychology, 63, 3-5.

Safran, J. D. \& Muran, J. C. (1996). The resolution of ruptures in the therapeutic alliance. Journal of Consulting and Clinical Psychology, 64, 447-458.

Santeiro, T. V. (2005). Psicoterapias breves psicodinâmicas: produção científica em periódicos nacionais e estrangeiros (1980/ 2002). Tese de Doutorado, Pontifícia Universidade Católica de Campinas, Campinas.
Seligman, M. E. P. (1996). Science as an ally of practice. American Psychologist, 51, 1072-1079.

Sexton, H. C., Hembre, K. \& Kvarme, G. (1996). The interaction of the alliance and therapy microprocess: a sequential analysis. Journal of Consulting and Clinical Psychology, 64, 471-480.

Sobell, M. B. \& Sobell, L. C. (2000). Stepped care as a heuristic approach to the treatment of alcohol problems. Journal of Consulting and Clinical Psychology, 68, 573-579.

Stilles, W. B. \& Shapiro, D. A. (1995). Verbal exchange structure of brief psychodynamic-interpersonal and cognitive-behavioral psychotherapy. Journal of Consulting and Clinical Psychology, 63, 15-27.

VandenBos, G. R. (1996). Outcome assessment of psychotherapy. American Psychologist, 51, 1005-1006.

Watson, J. C. (1996). The relationship between vivid description, emotional arousal, and in-session resolution of problematic reactions. Journal of Consulting and Clinical Psychology, 64, 459-464.

Wilson, G. T., Vitousek, K. M. \& Loeb, K. L. (2000). Stepped care treatment for eating disorders. Journal of Consulting and Clinical Psychology, 68, 564-572.

Witter, G. P. (1999). Apresentação. Em G. P. Witter (Org.), Produção científica em Psicologia e Educação (pp. 7-8). Campinas: Alínea. 